\title{
New data on spiders (Aranei) from the Novaya Zemlya Archipelago and Siberian Arctic, Russia
}

\author{
Новые данные по паукам (Aranei) архипемага Новая Земмя \\ и Сибирской Арктики, Россия
}

\begin{abstract}
A.V. Tanasevitch
A.B. Танасевич

A.N. Severtsov Institute of Ecology and Evolution, Russian Academy of Sciences, Leninsky prospekt 33, Moscow 119071 Russia. E-mail: tanasevitch@gmail.com

Институт проблем экологии и эволюции имени А.Н. Северцова РАН, Ленинский проспект 33, Москва 119071 Россия.
\end{abstract}

KEY WORDS: Russian Arctic, arctic archipelagoes, arctic islands, arctic tundra, new records.

КЛЮЧЕВЫЕ СЛОВА: Российская Арктика, арктические архипелаги, арктические острова, арктические тундры, новые находки.

ABSTRACT. New data on spiders from the Novaya Zemlya Archipelago are provided. A species list of the Bezymyannaya Bay, Southern Island of the Novaya Zemlya is added, and a few species from the earlier uninvestigated Krestovaya Bay, Northern Island are reported. Walckenaeria clavicornis (Emerton, 1882) is recorded from the Novaya Zemlya for the first time. Considering new data, the spider fauna of the Archipelago is currently known to contain 22 species. A few new records of spiders from different localities of the Siberian sector of Arctic are given.

How to cite this article: Tanasevitch A.V. 2018. New data on spiders (Aranei) from the Novaya Zemlya Archipelago and Siberian Arctic, Russia // Arthropoda Selecta. Vol.27. No.1. P.69-71. doi: 10.15298/arthsel. 27.1.10

РЕЗЮМЕ. Приведены новые данные по паукам архипелага Новая Земля. Список пауков бухты Безымянная (Южный о-в Новой Земли) дополнен; приведены находки пауков ранее неисследованной бухты Крестовая (Северный о-в). Вид Walckenaeria clavicornis (Emerton, 1882) впервые отмечен для фауны архипелага. С учётом новых данных, фауна пауков Новой Земли насчитывает 22 вида. Приведены также новые сведения о пауках из различных точек сибирского сектора Арктики.

\section{Introduction}

Two papers on Arctic spiders have recently been published: a revision of the spider fauna of the Novaya Zemlya [Tanasevitch, 2017b], and a annotated list of new records of spiders from different localities of the continental and insular territories of the Russian Arctic [Tanasevitch, 2017a]. A new portion of material from the Novaya Zemlya have been located in the collection of the Zoological Museum of the Moscow State University Moscow, Russia (ZMMU), as well as several earlier unaccounted samples collected from the Siberian Arctic by Yuri I. Chernov and Anatoly B. Babenko (Moscow, Russia).

A small material from the Novaya Zemlya was taken by Valery I. Bulavintsev (Moscow, Russia) from two localities of the Archipelago: from the Bezymyannaya Bay $\left(72.895^{\circ} \mathrm{N} 53.197^{\circ} \mathrm{E}\right)$, Southern Island, in 1992 , and from the Krestovaya Bay (ca $74.294^{\circ} \mathrm{N}$ $55.482^{\circ} \mathrm{E}$ ), Northern Island in 1993 . Only three spider species have hitherto been recorded from the Bezymyannaya Bay [see Tanasevitch, 2017b], none from the Krestovaya Bay.

The present paper provides new data on the spiders from the Novaya Zemlya and Siberian tundras.

\section{Material and methods}

The present paper is based on material kept at the ZMMU. Abbreviation in the text: NZ — Novaya Zemlya.

New data on spiders from the Novaya Zemlya Archipelago

Family LINYPHIIDAE

Agyneta nigripes (Simon, 1884)

Agyneta nigripes (?) — Tanasevitch, 2017: 148.

MATERIAL: $2 \sigma^{7} \sigma^{7}, 5$ o⿱, NZ, Southern Island, Bezymyannaya Bay, 1992, leg. V. Bulavintsev.

RECORDS FROM NZ: Southern Island: Bezymyannaya Bay and Matochkin Shar [see Tanasevitch, 2017]. From both above localities, $A$. nigripes has been identified, based on females alone, but in the absence of males, the determination could not be considered as reliable. The presence of males in the new material from Bezymyannaya Bay confirms the record of $A$. nigripes from the Archipelago. 
Diplocephalus barbiger (Roewer, 1955)

Diplocephalus barbiger — Tanasevitch, 2017: 148.

MATERIAL: 1 ㅇ, NZ, Northern Island, Krestovaya Bay, 1992 leg. V. Bulavintsev, new locality.

RECORDS FROM NZ: Southern Island: Malye Karmakuly; Bezymyannaya Bay; Cape Yuzhnyi Gusinyi Nos. Northern Island: Mashigina Bay; Blaafjell Island [Tanasevitch, 2017b].

\section{Halorates boreus (L. Koch, 1879)}

Halorates boreus — Tanasevitch, 2017: 149.

MATERIAL: $1 \sigma^{\top}, 1$, NZ, Southern Island, Bezymyannaya Bay, 1992, leg. V. Bulavintsev; 2 우, Northern Island, Krestovaya Bay, 1992, leg. V. Bulavintsev, new locality.

RECORDS FROM NZ: Bezymyannaya Bay [Tanasevitch, 2017b].

\section{Halorates holmgreni (Thorell, 1871)}

Halorates holmgreni — Tanasevitch, 2017: 149

MATERIAL: $5 \bigcirc^{7} \sigma^{7}, 31$ 우, NZ, Southern Island, Bezymyannaya Bay, 1992, leg. V.B; 4 90 , Northern Island, Krestovaya Bay, 1992, leg. V. Bulavintsev, new locality.

RECORDS FROM NZ: Southern Island: Pomorka Bay; Lichyutina Island; Cape Kostin Nos; Malye Karmakuly; Bezymyannaya Bay; Matochkin Shar Strait [Tanasevitch, 2017b].

\section{Halorates spetsbergensis (Thorell, 1872)}

Halorates spetsbergensis — Tanasevitch, 2017: 150.

MATERIAL: $2 \sigma^{7} \sigma^{7}, 5$ 오, NZ, Southern Island, Bezymyannaya Bay, 1992, leg. V. Bulavintsev, new locality; $7 \sigma^{\top} \sigma^{\top}, 10$ ㅇ, Northern Island, Krestovaya Bay, 1992, leg. V. Bulavintsev, new locality.

RECORDS FROM NZ: Southern Island: Cape Pomorka (Pomorskaya) Bay; Malye Karmakuly; Bezymyannaya Bay; Gribovaya Bay \& Vesyologo Island; Matochkin Shar Strait. Nothern Island: Tyuleniya Bay; Cape Sukhoi Nos; Tsyvolki Bay; Mashigina Bay; Blaafjell Island \& Mt Ditrihson; Admiralteystva Peninsula; Arkhangel'skaya Bay \& Lichyutina Island; Krestovye islands; Pankratieva Peninsula; Russkaya Gavan' Bay [Tanasevitch, 2017b].

\section{Hybauchenidium aquilonare (L. Koch, 1879)}

Hybauchenidium aquilonare - Tanasevitch, 2017: 150

MATERIAL: 1 q, NZ, Southern Island, Bezymyannaya Bay, 1992, leg. V. Bulavintsev, new locality.

RECORDS FROM NZ: Southern Island: Cape Kostin Nos; Cape Yuzhnyi Gusinyi Nos; Malye Karmakuly. Northern Island: Mashigina Bay; Blaafjell Island [Tanasevitch, $2017 \mathrm{~b}$.

\section{Mughiphantes sobrius (Thorell, 1871)}

Mughiphantes sobrius — Tanasevitch, 2017: 150.

MATERIAL: $6 \sigma^{\top} \sigma^{\top}, 10$ 우, NZ, Southern Island, Bezymyannaya Bay, 1992, leg. V. Bulavintsev, new locality.

RECORDS FROM NZ: Southern Island: Cape Pomorka (Pomorskaya) Bay; Cape Severnyi Gusinyi Nos; Bezymyannaya Bay; Matochkin Shar Strait. Northern Island: Lazareva Mts; Serebryanka Bay; Straumsen Bay; Arkhangel'skaya Bay; Lichyutina Island [Tanasevitch, 2017b].

\section{Walckenaeria clavicornis (Emerton, 1882)}

MATERIAL: 1 q, NZ, Southern Island, Bezymyannaya Bay, 1992, leg. V. Bulavintsev.
REMARKS: This species is new to the fauna of the Novaya Zemlya.

\section{New records of spiders in the Siberian Arctic}

After my recent publication [Tanasevitch, 2017a] another few samples from different localities of Siberia's Arctic have become available. Some new records are thus given below. If the species has already been known from the locality, then I give only the record(s) that derive from other habitat(s). At the same time, since most of the spider records from the Russian Arctic have not been attributed to any kind of habitat, e.g., Eskov [1985], Marusik et al. [1992, 1993], etc., any new habitat information concerning this hardly accessible region is significant.

\section{Family DICTYNIDAE}

\section{Dictyna major Menge, 1869}

MATERIAL: $1 \sigma^{r}, 1$, Yakutia Republic, Kolyma Bay, Kolyma River Delta, Pokhodskaya Yedoma $\left(69^{\circ} 32^{\prime} \mathrm{N} 160^{\circ} 44^{\prime} \mathrm{E}\right)$, meadow, sweeping, 18-19.VII.1994, leg. A. Babenko (R-45/94).

\section{Family LINYPHIIDAE}

\section{Erigone psychrophila Thorell, 1872}

MATERIAL: 11 오, Yakutia Republic, Laptev Sea coast, Olenyok Bay, Lake Vaganytta-Kyuel $\left(73^{\circ} 30^{\prime} \mathrm{N} 118^{\circ} 10^{\prime} \mathrm{E}\right)$, in wet depression, Dryas communities on slope, 6-8.VII.1994, leg. A. Babenko (R-12/94); $2 \sigma^{7} \sigma^{7}$, New Siberian Islands, Kotelnyi Island, Balyktakh River flow $\left(75^{\circ} 03^{\prime} \mathrm{N} 140^{\circ} 10^{\prime} \mathrm{E}\right)$, Bunge Land, in moss, 31.VII.-2.VIII.1994, leg. A. Babenko (R-62/94); $10^{7}, 1$, New Siberian Islands, Faddey Island, Ulakhan-Uryakh River flow $\left(75^{\circ} 35^{\prime} \mathrm{N} 144^{\circ} 50^{\prime} \mathrm{E}\right)$, in moss near puddle, pitfall traps, 10-11.VII. 1994, leg. A. Babenko (R-24/94); $1 \sigma^{\top}, 1$ ㅇ, same date and locality, slope of bank, pitfall traps, 10-11.VII.1994, leg. A. Babenko (R26/94).

\section{Halorates holmgreni (Thorell, 1871)}

MATERIAL: 3 우, Yakutia Republic, Kolyma Bay, Kolyma River Delta, Pokhodskaya Yedoma $\left(69^{\circ} 32^{\prime} \mathrm{N} 160^{\circ} 44^{\prime} \mathrm{E}\right)$, meadow, sweeping, 18-19.VII.1994, leg. A. Babenko (R-45/94).

\section{Halorates spetsbergensis (Thorell, 1872)}

MATERIAL: 1 , western Taimyr Peninsula, Byrranga Mts, Lake Kosoturku (ca $73.188^{\circ} \mathrm{N} 83.4795^{\circ}$ ), near spring, pitfall traps, 3.VIII.1986, leg. Yu. Chernov; $1 \bigcirc^{\top}$, New Siberian Islands, Kotelnyi Island, Balyktakh River flow $\left(75^{\circ} 03^{\prime} \mathrm{N} 140^{\circ} 10^{\prime} \mathrm{E}\right)$, sedge swamp on low river terrace, 31.VII-2.VIII.1994, leg. A. Babenko (R-58/ 94); 1 , New Siberian Islands, Faddey Island, Ulakhan-Uryakh River flow $\left(75^{\circ} 35^{\prime} \mathrm{N} 144^{\circ} 50^{\prime} \mathrm{E}\right)$, hillock with Salix polaris and miscellaneous herbs, 10-11.VII.1994, leg. A. Babenko (R-17/94).

\section{Mughiphantes sobrius (Thorell, 1872)}

MATERIAL: 1 , Krasnoyarsk Area, Taymyrsky DolganoNenetsky District, western Taymyr Peninsula, Ragozinka River flow, spotted tundra, 20.VI.1990, leg. Yu. Chernov.

\section{Semljicola arcticus (Eskov, 1989)}

MATERIAL: 5 우, Krasnoyarsk Area, Taymyrsky DolganoNenetsky District, NW Taymyr Peninsula, Uboynaya River flow, zonal tundra, pitfall traps, 21.VIII.1988, leg. Yu. Chernov. 
Semljicola barbiger (L. Koch, 1879)

MATERIAL: 1 q, Krasnoyarsk Area, Taymyrsky DolganoNenetsky District, NW Taymyr Peninsula, Ragozinka River flow, landslide, bare ground, 8.VIII.1993, leg. Yu. Chernov.

\section{Family LYCOSIDAE}

Alopecosa mutabilis (Kulczyński, 1908)

MATERIAL: 1 \%, Yakutia Republic, East Siberian Sea coast, Indigirka River Delta $\left(71^{\circ} 26^{\prime} \mathrm{N} 149^{\circ} 45^{\prime} \mathrm{E}\right)$, Russko-Ustinskaya River branch, spotted tundra on top of hill, 14-16.VII.1994, leg. A Babenko (R-33/94); 2 क⿱ , Kolyma Bay, Kolyma River Delta, Pokhodskaya Yedoma $\left(69^{\circ} 32^{\prime} \mathrm{N} 160^{\circ} 44^{\prime} \mathrm{E}\right)$, slope with willows, dwarf birch and boreal shrubs/undershrubs, 18-19.VII.1994, leg. A. Babenko (R-40/94)

\section{Pardosa algens (Kulczyński, 1908)}

MATERIAL: 1 + , Yakutia Republic, Kolyma Bay, Kolyma River Delta, Pokhodskaya Yedoma $\left(69^{\circ} 32^{\prime} \mathrm{N} 160^{\circ} 44^{\prime} \mathrm{E}\right)$, under stones at foot of permafrost hummock, 18-19.VII.1994, leg. A. Babenko (R-48/94); $1 \sigma^{7}$, same date and locality, slope with dwarf willows and birch, pitfall traps, leg. A. Babenko (R-50/94).

\section{Family THOMISIDAE}

\section{Xysticus albidus Grese, 1909}

MATERIAL: $1 \sigma^{7}, 1$, Yakutia Republic, Kolyma Bay, Kolyma River Delta, Pokhodskaya Yedoma $\left(69^{\circ} 32^{\prime} \mathrm{N} 160^{\circ} 44^{\prime} \mathrm{E}\right)$, meadow, sweeping, 18-19.VII.1994, leg. A. Babenko (R-45/94).
ACKNOWLEDGEMENTS. I am most grateful to all collectors whose material has been used in this work. Thanks go also to Kirill G. Mikhailov (Moscow) for the opportunity to work with museum collections under his care, and to Sergei I. Golovatch (Moscow) who kindly checked the English of an early draft. The study was funded in part by the Russian Foundation for Basic Research, research project \#15-04-05964 A.

\section{References}

Eskov K.Yu. 1985. [Spiders of the tundra zone of the USSR] // Ovcharenko V.I. (ed.). Faina i ekologiya paukov SSSR. Trudy Zool. Inst. AN SSSR, Leningrad. Vol.139. P.121-128 [in Russian].

Marusik Yu.M., Eskov K.Y., Kim J.P. 1992. A check list of spiders (Aranei) of Northeast Asia // Korean Arachnol. Vol.8. Nos 1/2. P.129-158.

Marusik Yu.M., Eskov K.Yu., Koponen S., Vinokurov N.N. 1993. A check-list of the spiders (Aranei) of Yakutia, Siberia // Arthropoda Selecta. Vol.2. No.2. P.63-79.

Tanasevitch A.V. 2017a. New records of spiders (Aranei) from the Russian Arctic // Arthropoda Selecta. Vol.26. No.1. P.77-82.

Tanasevitch A.V. 2017b. Spiders (Aranei) of the Novaya Zemlya Archipelago and the Vaygach Island, Russia // Arthropoda Selecta. Vol.26. No.2. P.145-153.

Responsible editor K.G. Mikhailov 